\title{
Prospective control study of efficacy and influencing factors of a ketogenic diet on refractory epilepsy in children
}

\author{
Lianying Feng^, Juan Wang, Xiujuan Li, Yue Hu, Siqi Hong, Li Jiang \\ Department of Pediatric Neurology, Children's Hospital of Chongqing Medical University, National Clinical Research Center for Child Health and \\ Disorder, Ministry of Education Key Laboratory of Child Development and Disorders, Chongqing Key Laboratory of Pediatrics, Chongqing Key \\ Laboratory of Translational Medical Research in Cognitive Development and Learning and Memory Disorders, Chongqing, China \\ Contributions: (I) Conception and design: L Feng, J Wang, L Jiang; (II) Administrative support: S Hong, L Jiang; (III) Provision of study materials or \\ patients: J Wang, X Li, Y Hu, S Hong; (IV) Collection and assembly of data: L Feng, J Wang; (V) Data analysis and interpretation: All authors; (VI) \\ Manuscript writing: All authors; (VII) Final approval of manuscript: All authors. \\ Correspondence to: Li Jiang. Department of Pediatric Neurology, Children's Hospital of Chongqing Medical University, National Clinical Research \\ Center for Child Health and Disorder, Ministry of Education Key Laboratory of Child Development and Disorders, Chongqing Key Laboratory \\ of Pediatrics, Chongqing Key Laboratory of Translational Medical Research in Cognitive Development and Learning and Memory Disorders, \\ Chongqing, China. Email: jiang1666999@163.com.
}

Background: To investigate the efficacy of the ketogenic diet (KD) and anti-epileptic drugs (AEDs) on children with refractory epilepsy (RE), and to analyze the effects of gender, therapeutic time, age, type of epilepsy syndrome, number of epileptic seizure types, and number of AEDs on the efficacy of the KD. A prospective, non-randomized, controlled cohort study was used and a total of 200 children with RE who were hospitalized in the Department of Pediatric Neurology, Children's Hospital of Chongqing Medical University from November 2015 to December 2020 were divided into a KD group and AEDs only group according to their parents' wishes.

Methods: Children in the KD group were treated with a classical KD in addition to the AEDs, while in the AEDs group, the original AEDs were continued and could be adjusted according to the clinical seizure types and other conditions. The effects of gender, therapeutic time, age, type of epilepsy syndrome, number of epileptic seizure types, and number of AEDs on the efficacy of the KD were analyzed.

Results: In the KD group, the efficacy was $79.41 \%, 79.59 \%, 81.05 \%, 81.11 \%, 77.01 \%$, and $78.75 \%$, at $3,6,9,12,18$, and 24 months, respectively. In the AEDs group, the efficacy was $59.18 \%, 60.42 \%, 59.78 \%$, $59.55 \%, 59.30 \%$, and $53.01 \%$ at 3, 6, 9, 12, 18, and 24 months, respectively. After 3, 6, 9, 12, 18, and 24 months of treatment, a statistically significant difference in the effective rate between the KD group and the AEDs group was seen $(\mathrm{P}<0.05)$. Chi-square test was used to analyze the effects of gender, therapeutic time, age, type of epilepsy syndrome, number of epileptic seizure types, and number of AEDs on the efficacy of the KD, and the results showed no statistical significance $(\mathrm{P}>0.05)$.

Conclusions: KD therapy reduces the frequency of seizures in children with RE compared with AEDs alone. In the KD group, the gender, therapeutic time, age, type of epilepsy syndrome, number of epileptic seizure types, and number of anti-epileptic drugs had no significant effect on the efficacy of the KD diet.

Keywords: Ketogenic diet; children; refractory epilepsy; efficiency; influence factors

Submitted Nov 26, 2021. Accepted for publication Jan 17, 2022.

doi: $10.21037 / \mathrm{tp}-21-595$

View this article at: https://dx.doi.org/10.21037/tp-21-595

^ ORCID: 0000-0001-5464-693X. 


\section{Introduction}

Epilepsy is a heavy burden on children, who can have changes in behavior or movement due to repeated abnormal electrical discharges in the brain (1). Epilepsy as one of the most common neurological diseases, with a prevalence in children of $0.3-0.6 \%$ (2). While drug therapy is currently the preferred treatment, $20-30 \%$ of children develop drug-refractory epilepsy [referred to as refractory epilepsy (RE)] (2). For refractory epilepsy, we can choose ketogenic diet $(\mathrm{KD})$, vagal nerve stimulation or surgery for different causes. The ketogenic diet was developed in 1921 and consists of high fat, moderate protein, and low carbohydrates. In the 1920s and 1930s, ketogenic diets were used to treat epilepsy, but the emergence of new anti-epileptic drugs (AEDs) made AEDs the dominant antiepileptic treatment (3). Because AEDs have fewer side effects and are more convenient than ketogenic diet. Ketogenic diets returned to the public consciousness in the 1990s after the publication of a promising study that cured a 2-year-old children with epilepsy with a ketogenic diet, and the founding of the Charlie Foundation, a nonprofit that provides dietary therapy for people with epilepsy (4). However, many neurologists are not familiar with the use of ketogenic diets. In the past few years, alternative and more flexible $\mathrm{KD}$ variants to the classic form have been developed, including a medium-chain triglyceride diet, modified Atkins diet, and low glycemic index treatment (5). The use of ketogenic diet needs the joint participation of clinicians and dietitians, so that more patients with refractory epilepsy can get effective treatment. Clinical studies have shown that the $\mathrm{KD}$ can reduce seizure frequency in about half of children with RE by more than $50 \%$ (6-8). However, the anti-epileptic mechanisms of the $\mathrm{KD}$ and related factors that affect therapeutic outcomes remain unclear. A large number of ketone bodies (acetone, acetoacetic acid and $\beta$-hydroxybutyric acid) produced during $\mathrm{KD}$ treatment can affect sodium and potassium ion channels, cause neuronal depolarization and reduce neuronal excitability, thus playing an anti-epileptic role. KD can increase the synthesis of polyunsaturated fatty acids in vivo, and the latter can inhibit the voltage-gated sodium channel in neurons, thus exerting its antiepileptic effect. In recent years, it has been found that KD can inhibit the activation of mammalian target of rapamycin (mTOR) in hippocampus, thus playing an anti-epileptic role. KD can play an anti-epileptic role by activating the adenosine A1 receptor, which in turn enables the latter to regulate adenosine kinase (9). There are very few prospective controlled studies on the $\mathrm{KD}$ in the treatment of RE in children in southwest China, and a prospective non-randomized controlled cohort study was conducted in this study. In this study, we investigated the effects of multiple factors on the ketogenic diet and confirmed its effectiveness in treating refractory epilepsy. As is known to all, Chongqing is located in the west of China. It is not easy to carry out ketogenic diet in the relatively backward areas of western China. This study can bring hope to more children with refractory epilepsy in western China and guide the development of ketogenic diet in other neighboring cities of western China. We present the following article in accordance with the STROBE reporting checklist (available at https://tp.amegroups.com/article/ view/10.21037/tp-21-595/rc).

\section{Methods}

\section{Subjects}

A prospective non-randomized controlled cohort study was used in this study and was approved by the Ethics Committee of the Children's Hospital of Chongqing Medical University [Approval document No. (2017) ethics review (research) No. 122]. All procedures performed in this study involving human participants were in accordance with the Declaration of Helsinki (as revised in 2013) and informed consent was taken from the patients' guardians. A total of 200 children with RE who were hospitalized in the Department of Pediatric Neurology, Children's Hospital of Chongqing Medical University from November 2015 to December 2020 were divided into a KD treatment group and AEDs only group according to their parents' wishes. Inclusion criteria were as follows: (I) all children met the diagnostic criteria for drug-RE, which states that seizures cannot be completely controlled after the use of two or more AEDs with a reasonable and sufficient amount of single drug or combination therapy (International League Against Epilepsy (ILAE) 2010) (2). (II) Patients could not have previously received KD treatment. (III) Family members agreed to KD treatment and provided written informed consent. Exclusion criteria were as follows: (I) absolute contraindications to $\mathrm{KD}$ treatment, such as fatty acid oxidation defects, pyruvate carboxylase deficiency, glycogen storage disease (except type 2), ketone generated defects, ketone metabolic disorders, porphyrins, long QT syndrome or other heart disease, liver, kidney or pancreatic insufficiency, high insulin hematic disease, and being less 
than 2 months of age (7). (II) Poor compliance or the presence of related factors that affect treatment compliance, such as serious heart, lung, and blood system diseases; liver and kidney function impairment; hyperlipidemia; urinary calculi; or severe gastrointestinal reflux. In the $\mathrm{KD}$ group, 102 children were enrolled, and 102, 98, 95, 90, 87, and 80 children persisted in treatment for $3,6,9,12,18$, and 24 months, respectively. In the AEDs group, 98 children were enrolled, and 98, 96, 92, 89, 86, and 83 children persisted in treatment for $3,6,9,12,18$, and 24 months, respectively.

\section{Study methods}

We used the classical KD in addition to AEDs in the KD group. Laboratory assessment prior to the start of the diet, included blood tandem mass spectrometry, urine gas chromatography, blood routine, urine routine and urine calcium, liver and kidney function, electrolyte, blood sugar, blood gas analysis, trace element, fasting blood lipid, concentration of AEDs, abdominal ultrasound, urinary tract ultrasound, echocardiogram and cardiac function, chest $\mathrm{X}$-rays, bone age and bone mineral density, intelligence assessment, cranial MRI, and EEG examination, and when it is was necessary, a gene examination was performed (10).

Doctors and nutritionists jointly integrated the basic level of children's growth and development, activity level, standard weight, and nutritional status to construct the diet plan. The KD commenced at $1 / 3$ of the total amount on the first day, 2/3 of the total amount on the second day, and the full amount on the third day. A variety of vitamins, minerals and calcium and potassium were added during the $\mathrm{KD}$, and children could drink freely. Blood glucose and blood ketone bodies were monitored every 8 hours, and if the former $<2.2 \mathrm{mmol} / \mathrm{L}, 2-4$ gram carbohydrates were given orally, and the level reviewed 15-20 minutes later. Blood gas analysis was performed if the blood ketone was more than $5 \mathrm{mmol} / \mathrm{L}$. The type and dose of AEDs were not adjusted $(11,12)$ during the diet, and the hospital stay during the diet was for one week. Members of the KD group were then discharged after ketogenic bodies were stabilized by adjusting eating methods and time, and the diet continued outside the hospital.

Children in the AEDs group continued their original anti-epileptic drugs therapy after enrollment although this was continuously monitored and modified subject to the type of seizure, age, severity of seizure, diagnosis of a specific epilepsy syndrome, the cause of the epilepsy, and comorbidities, when appropriate.

It is suggested that if patients insist on $\mathrm{KD}$ treatment for 3 months, but the attack decrease less than $50 \%$ can be considered as invalid. If the seizures are completely controlled for two years, according to the clinical situation, it is generally considered to stop KD (10). For patients with complete seizure control, electroencephalogram examination and clinical data evaluation should be performed before KD discontinuation. Inform parents of the risk of recurrence after $\mathrm{KD}$ discontinuation. During $\mathrm{KD}$ transition, $\mathrm{KD}$ is usually phased out unless it is necessary to stop KD urgently.

The main factors affecting compliance were efficacy, side effects, economic factors and ketogenic diet taste. If the children have adverse reactions, we will deal with them in time, and actively communicate with the families of the children to relieve their anxiety and increase their compliance.

\section{Evaluation of therapeutic effect}

Evaluation of the therapeutic effect was determined by the frequency of seizures 3 months before enrollment and 3, 6, 9, 12, 18, and 24 months after treatment, and compared between the two groups. Parents of the enrolled children recorded the frequency of seizures before and after enrollment for statistical analysis. However, since the parents are not professional pediatric neurologists, there is information bias in the judgment of the children's seizure type, seizure time, seizure frequency, and other information. To help solve this and more accurately record details, an education program was provided to parents detailing how to identify when a seizure begins, the different types of seizures, how to perform first aid after seizures, and common problems associated with the use of AEDs. The Engel grading criteria (13-15) was used to evaluate the reduction in seizure frequency in children treated receiving the KD and AEDs continuously for 3, 6, 9, 12, 18, or 24 months. The Engel classification includes the following four levels: Grade I no seizures; Grade II more than 90\% reduction in seizure frequency; Grade III 50-90\% reduction in seizure frequency; Grade IV less than $50 \%$ reduction in seizure frequency. The efficacy rate $=$ (total number of grade I + II + III)/all the enrolled children $\times 100 \%$.

\section{Statistical methods}

The number of cases during the study period determined 
Table 1 Clinical features of enrolled patients

\begin{tabular}{|c|c|c|c|c|c|}
\hline Groups & Gender & $\begin{array}{l}\text { Average therapeutic } \\
\text { time (month) }\end{array}$ & $\begin{array}{l}\text { Average age } \\
\text { (months) }\end{array}$ & Epilepsy syndrome & Seizure types \\
\hline KD group & $\begin{array}{l}\text { Male: } 54 \text {, } \\
\text { Female: } 48\end{array}$ & $21.15 \pm 6.07$ & $36.17 \pm 39.74$ & IS: 45; LGS: 13; FIRES: 7 & $\begin{array}{c}1 \text { type: } 40 ; 2 \text { type: } 35 ; 3 \text { type: } 18 \text {; } \\
4 \text { type: } 5 ; 5 \text { type: } 3 ; 6 \text { type: } 1\end{array}$ \\
\hline $\begin{array}{l}\text { AEDs } \\
\text { group }\end{array}$ & $\begin{array}{l}\text { Male: } 52 \text {, } \\
\text { Female: } 46\end{array}$ & $21.83 \pm 5.50$ & $34.14 \pm 36.57$ & IS: 38; LGS: 9; FIRES: 4; DS: 5 & $\begin{array}{c}1 \text { type: } 35 \text {; } 2 \text { type: } 29 ; 3 \text { type: } 17 \text {; } \\
4 \text { type: } 9 ; 5 \text { type: } 5 \text {; } 6 \text { type: } 3\end{array}$ \\
\hline
\end{tabular}

$\mathrm{KD}$, ketogenic diet; AEDs, anti-epileptic drugs.

Table 2 Efficacy of refractory epilepsy in the ketogenic group

\begin{tabular}{lccccc}
\hline \multirow{2}{*}{ Therapeutic time (months) } & Total cases & \multicolumn{3}{c}{ Valid cases (\%) } & Invalid cases (\%) \\
\cline { 3 - 5 } & & Grade I (\%) & Grade I, II and III & Effective rate (\%) \\
\hline 3 & 102 & $61(59.80)$ & 20 & $81(79.41)$ & $21(20.59)$ \\
6 & 98 & $56(57.14)$ & 22 & $78(79.59)$ & $77(81.05)$ \\
12 & 95 & $53(55.79)$ & $20(20.41)$ & $73(81.11)$ & $18(18.95)$ \\
18 & 90 & $48(53.33)$ & $25(18.89)$ & $67(77.01)$ & $63(78.75)$ \\
\hline
\end{tabular}

the sample size, and children who dropped out of the study were no longer included in the efficacy analysis. SPSS 22.0 was used for data processing. Chi-square test was used to compare the treatment efficiency of the KD group and the AEDs group at different treatment times and analyze the effects of gender, therapeutic time, age, type of epilepsy syndrome, number of epileptic seizure types, and number of AEDs. Fisher's exact probability method was used to analyze those that did not meet the chi-square test conditions, and a $P$ value less than 0.05 was considered statistically significant.

\section{Results}

\section{Overview of clinical efficacy}

The profiles of all 200 cases are shown in Table 1. Three epileptic syndrome types were identified in the KD group: infantile spasm (IS), Lennox-Gastaut syndrome (LGS), and febrile infection-related epilepsy syndrome (FIRES), and four epileptic syndrome types were identified in the AEDs group: infantile spasm (IS), Lennox-Gastaut syndrome (LGS), febrile infection-related epilepsy syndrome (FIRES), and dravet syndrome (DS). In the KD group, 102, 98, 95, 90,87 , and 80 children were followed up to $3,6,9,12,18$, and 24 months, respectively. In the AEDs group, 98, 96, 92,
89,86 , and 83 children were followed up to $3,6,9,12,18$, and 24 months, respectively. Children dropped out of the study mainly because of poor outcomes. The therapeutic effects of the $\mathrm{KD}$ at 3, 6, 9, 12, 18, and 24 months are summarized in Table 2, and show the efficacy was $79.41 \%$, $79.59 \%, 81.05 \%, 81.11 \%, 77.01 \%$, and $78.75 \%$, and the total control rates were $59.80 \%, 57.14 \%, 55.79 \%, 53.33 \%$, $56.32 \%$, and $53.75 \%$ at $3,6,9,12,18$, and 24 months, respectively. The efficacy of the AEDs group only at 3, 6, 9, 12, 18, and 24 months are summarized in Table 3, and show this was $59.18 \%, 60.42 \%, 59.78 \%, 59.55 \%, 59.30 \%$, and $53.01 \%$, and the total control rates were $35.71 \%, 37.50 \%$, $32.61 \%, 32.58 \%, 32.56 \%$, and $28.92 \%$ at $3,6,9,12,18$, and 24 months, respectively.

\section{Comparison of efficacy between the KD group and AEDs group}

The efficacy of the KD group and the AEDs group are shown in Tables 2,3, and using chi-square test to compare the treatment efficacy of the two groups, as shown in Table 4. After 3, 6, 9, 12, 18, and 24 months of treatment, the comparison of efficacy of the two groups showed that the $\mathrm{P}$ value was less than 0.05 , confirming the efficacy of 
Table 3 Efficacy of refractory epilepsy in the AEDs group

\begin{tabular}{|c|c|c|c|c|c|}
\hline Therapeutic time (months) & Total cases & \multicolumn{3}{|c|}{ Valid cases $(\%)$} & Invalid cases $(\%)$ \\
\hline 3 & 98 & 35 (35.71) & 23 & $58(59.18)$ & $40(40.82)$ \\
\hline 6 & 96 & $36(37.50)$ & 22 & $58(60.42)$ & $38(39.58)$ \\
\hline 9 & 92 & $30(32.61)$ & 25 & $55(59.78)$ & $37(40.22)$ \\
\hline 18 & 86 & $28(32.56)$ & 23 & $51(59.30)$ & $35(40.70)$ \\
\hline 24 & 83 & 24 (28.92) & 20 & $44(53.01)$ & $39(46.99)$ \\
\hline
\end{tabular}

the KD was better than that of using AEDs only therapy.

\section{Analysis of factors influencing efficacy of the ketogenic diet}

Effect of gender on the efficacy of the ketogenic diet

The results showed that gender had no significant effect on the efficacy of the KD (Table 5).

\section{Effect of therapeutic time on efficacy of the ketogenic diet}

$102,98,95,90,87$, and 80 of the 102 children with RE were treated with the $\mathrm{KD}$ for 3, 6, 12, 18, and 24 months, respectively, and the effect of therapeutic time on the curative effect of the KD. The results in Table 6 show there is no significant effect of the therapeutic time on the efficacy of the KD.

\section{Effect of age on efficacy of the ketogenic diet}

Patients enrolled were divided into a 1 year or younger age group, 1-3 years of age, and $>3$ years of age, and the KD treatment efficacy ranged from $73.91-88.46 \%$. The results in Table 7 show that age had no significant effect on the effect of the KD.

\section{Effects of epilepsy syndrome types on efficacy of the ketogenic diet} Epilepsy types observed in the KD group were IS, LGS, and FIRES, and the efficacy of the KD ranged from $77.78 \%$ to $100 \%$, as shown in Table 8 . The results show that the type of epilepsy syndrome had no significant effect on the efficacy of the ketogenic diet

Effect of number of epileptic seizure types on efficacy of the ketogenic diet

We identified six forms of seizures, and KD treatment efficacy ranged from $68.42 \%$ to $85.71 \%$. Epileptic seizure types were divided into three groups: single type, two types, and $\geq 3$ types, and the results are summarized in Table 9. The results show that the number of epileptic seizure types had no significant effect on the efficacy of the KD.

\section{Effect of the number of anti-epileptic drugs on efficacy} of the ketogenic diet

Between two and five types of anti-epileptic medication were used during this study. Subjects were divided into two groups: $\leq$ three kinds of AEDs and > three kinds of AEDs, and the results in Table 10 show the number of AEDs had no significant effect on the efficacy of the KD.

\section{Discussion}

Wilder first used the KD to treat RE in 1921, and this treatment strategy has been widely used in the past 10 years. Retrospective, multi-center studies, and randomized controlled trials have demonstrated the effectiveness of KD therapy for treatment of RE (16-18).

One study of 216 children with RE treated with the KD followed up for 6 years and showed $56.5 \%$ experienced a sustained reduction in seizures greater than $75 \%$ (15). In 2008, Neal et al. (18) designed a randomized controlled trial to demonstrate the therapeutic effect of the KD in children with intractable epilepsy on 145 cases of children aged 2-16. The results showed that $38 \%$ of children had a greater than $50 \%$ reduction in epileptic seizure frequency, while only $6 \%$ of the control group had a similar reduction. This study also demonstrated that the therapeutic effect of $\mathrm{KD}$ therapy was similar in patients with comprehensive seizures and focal seizures. In 2016, a randomized controlled trial by Lambrechts et al. (19) showed that the efficacy of the KD was greater than $50 \%$ for treatment of children with RE. 


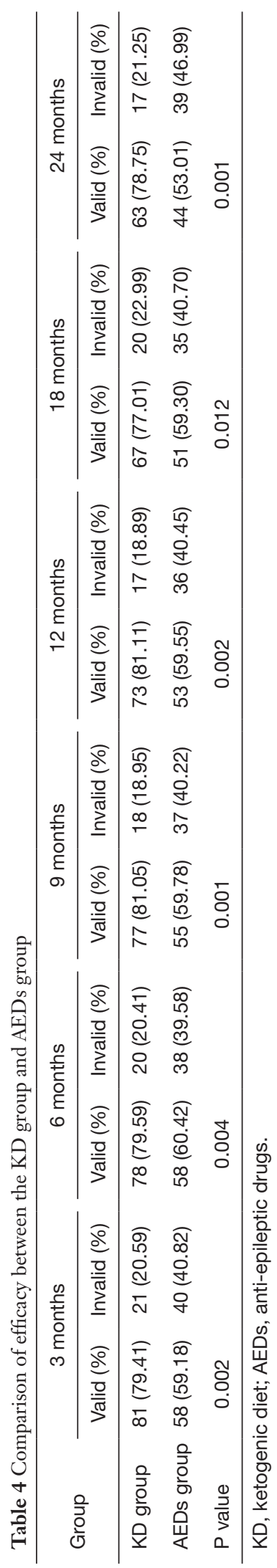

The KD results in energy production derived primarily from fat metabolism, with the $\beta$-oxidation of fatty acids as the main energy metabolism pathway in the body, resulting in systemic changes in metabolic products such as increased ketones $(20,21)$. The mechanism of the KD for the treatment of epilepsy is not clear, and factors associated with the efficacy of the KD treatment of epilepsy have not been determined. Therefore, this study evaluated the therapeutic effect of the KD in children with intractable epilepsy and analyzed associated factors, to facilitate the screening of children who are suitable for the diet, and to identify factors that that may improve its efficacy.

In this study, 200 children with RE were recruited. In the KD group, the efficacy was $79.41 \%, 79.59 \%, 81.05 \%$, $81.11 \%, 77.01 \%$, and $78.75 \%$, and the total control rates were $59.80 \%, 57.14 \%, 55.79 \%, 53.33 \%, 56.32 \%$, and $53.75 \%$ at $3,6,9,12,18$, and 24 months, respectively. In the AEDs group, the efficacy was $59.18 \%, 60.42 \%, 59.78 \%$, $59.55 \%, 59.30 \%$, and $53.01 \%$, and the total control rates were $35.71 \%, 37.50 \%, 32.61 \%, 32.58 \%, 32.56 \%$, and $28.92 \%$ at $3,6,9,12,18$, and 24 months, respectively. We used the chi-square test to compare the treatment efficacy of the two groups after 3, 6, 9, 12, 18, and 24 months of treatment, and the comparison showed that the $\mathrm{P}$ value was less than 0.05 , confirming the efficacy of the $\mathrm{KD}$ was better than that of using AEDs only therapy. This shows KD therapy is an effective treatment for children with RE.

The study showed that the efficacy of the KD was independent of gender and age, which is in agreement with the findings of a study by Kossoff et al. (16). However, the causes of epilepsy and treatment compliance in children in different age groups differ. Therefore, in future studies larger sample sizes will be necessary to further analyze the relationship between the efficacy of the KD and age distribution.

We found there was no significant effect of therapeutic time on the efficacy of the $\mathrm{KD}$, with efficacy ranging from $77.01 \%$ to $81.11 \%$ at all time points. Therefore, children who responded positively to the diet at 3 months should continue to follow it. However, whether children who respond to the KD should be treated for 24 months before stopping it is unclear. Kang et al. (22) conducted a study on the prognoses of children with IS treated with the KD for 6 months and those without epileptic seizures and found there was no statistical difference in prognosis or recurrence rate between the two groups of children treated for 8 months and 2 years. As a prolonged KD may affect normal growth and development, a reduction in the 
Table 5 Effect of gender on the efficacy of the ketogenic diet

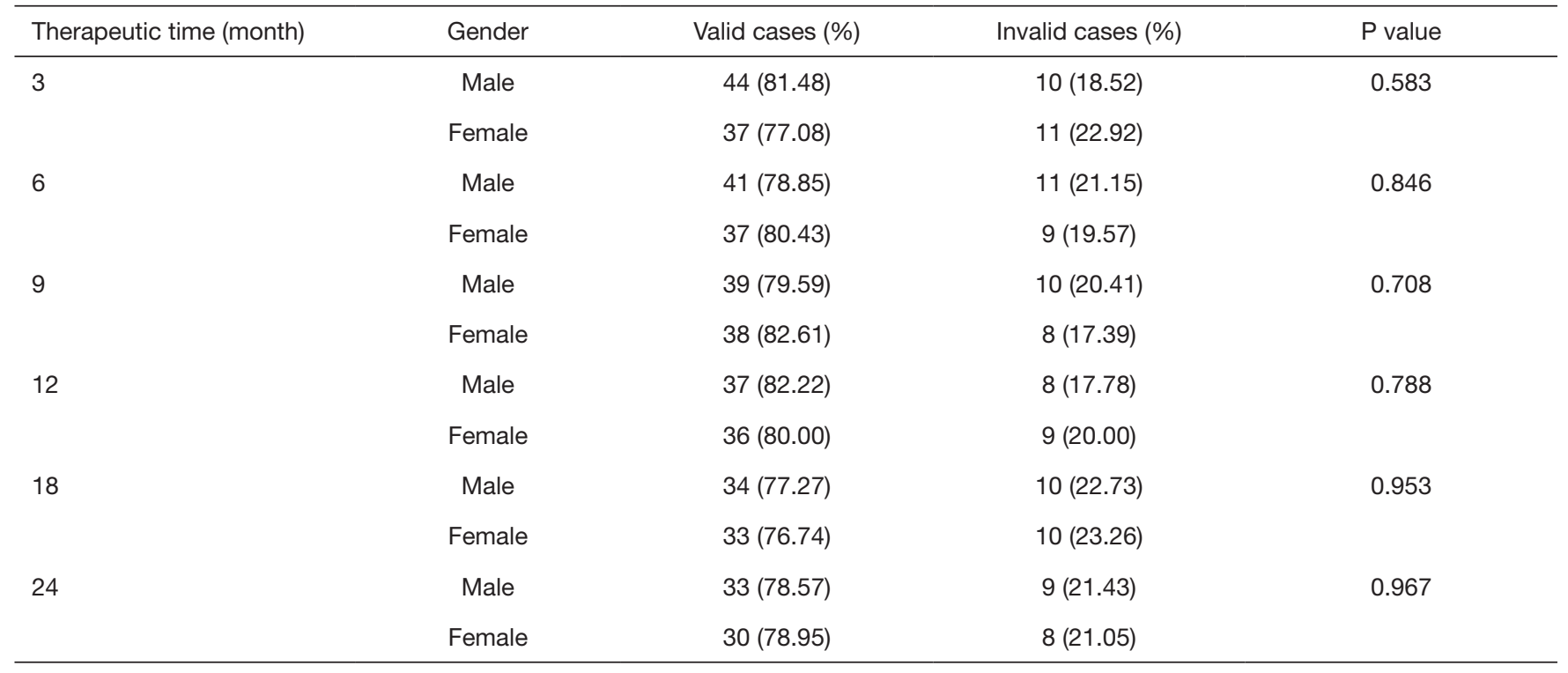

Table 6 Effect of therapeutic time on efficacy of the ketogenic diet

\begin{tabular}{lcc}
\hline Therapeutic time (month) & Valid cases $(\%)$ & Invalid cases $(\%)$ \\
\hline 3 & $81(79.41)$ & $21(20.59)$ \\
6 & $78(79.59)$ & $20(20.41)$ \\
9 & $77(81.05)$ & $18(18.95)$ \\
12 & $73(81.11)$ & $17(18.89)$ \\
18 & $67(77.01)$ & $20(22.99)$ \\
24 & $63(78.75)$ & $17(21.25)$ \\
\hline
\end{tabular}

treatment duration of children with IS should be considered in the absence of seizures.

Our study found that the number of epileptic seizure types had no significant effect on the efficacy of the KD. However, we did not evaluate associations between KD efficacy and specific seizure types due to sample size limitations. A retrospective, multi-center study by Caraballo et al. (17) showed that the KD was more effective for myoclonic seizures. Therefore, further studies are needed to evaluate the correlation of KD efficacy and seizure type. We also found that the efficacy rates of the $\mathrm{KD}$ for IS, LGS, and FIRES were between $77.78-100 \%$, but the results showed that the type of epilepsy syndrome had no significant effect on the efficacy of the diet However, Dhamija et al. (23) showed that the KD was very effective for the treatment of children with IS and those with Dravet syndrome and Landau-Kleffner syndrome (LKS). They suggested the KD could be used early in children with Dravet syndrome, rather than as a last resort when other treatments have failed. Kossoff et al. (16) showed differences in the efficacy of the KD among different types of epilepsy, and it was more effective in children with Dravet syndrome and LKS. A retrospective, multicenter study by Caraballo et al. (17) showed that the effectiveness of the KD followed the rank order of LGS >IS > Dravet syndrome.

The results showed that the number of AEDs had no significant effect on the efficacy of the KD. Therefore, the number of AEDs used should not limit use of the KD. However, an interaction between AEDs and the KD has been demonstrated. Spilioti et al. (24) showed that valproic acid affected the levels of ketones in the body, and van der Louw et al. (25) found that using lamotrigine was associated 
Table 7 Effect of age on the efficacy of the ketogenic diet

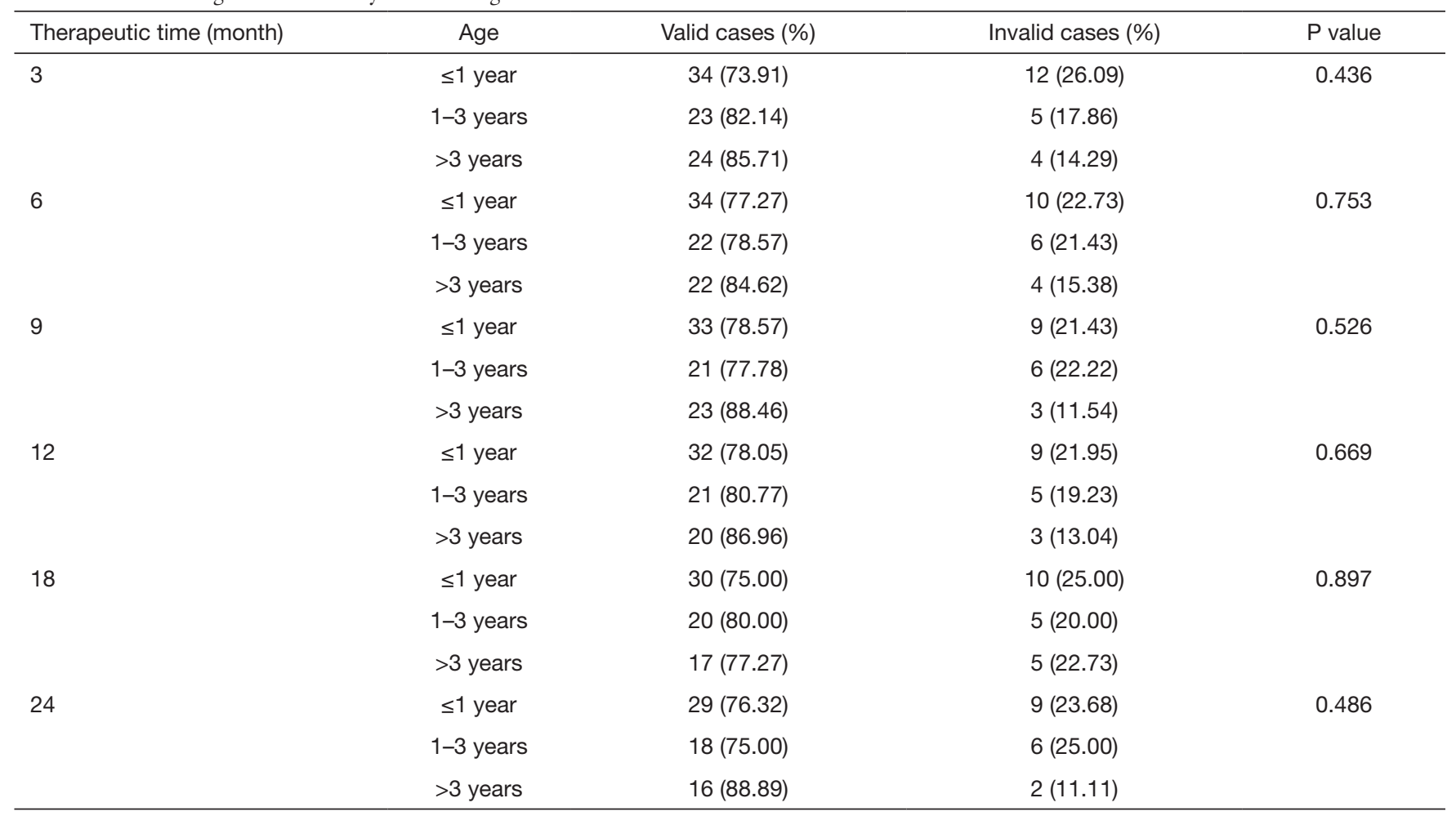

Table 8 Effects of epilepsy syndrome types on efficacy of the ketogenic diet

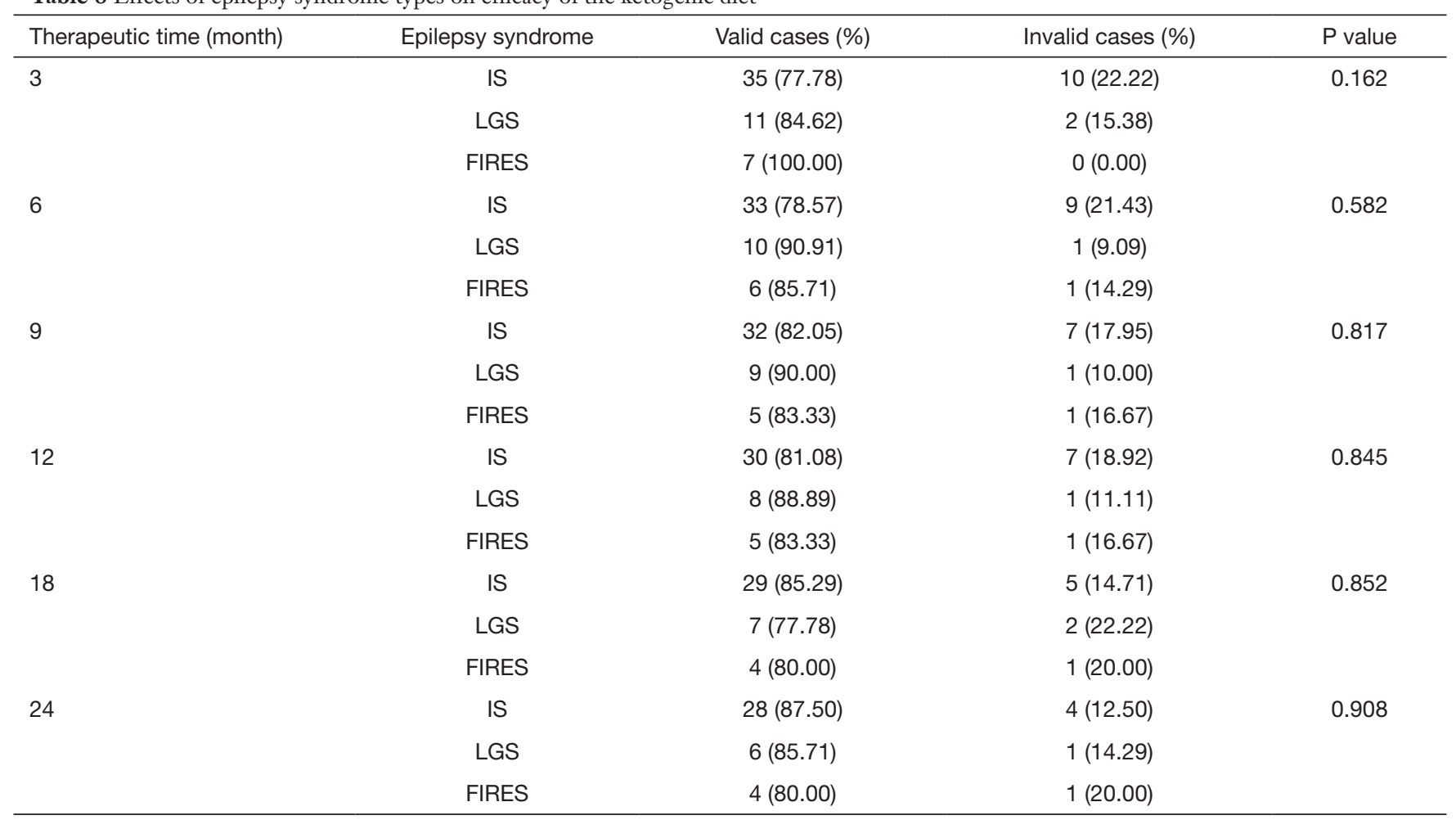

IS, infantile spasm; LGS, Lennox-Gastaut syndrome; FIRES, febrile infection-related epilepsy syndrome. 
Table 9 Effect of epileptic seizure type on efficacy of the ketogenic diet

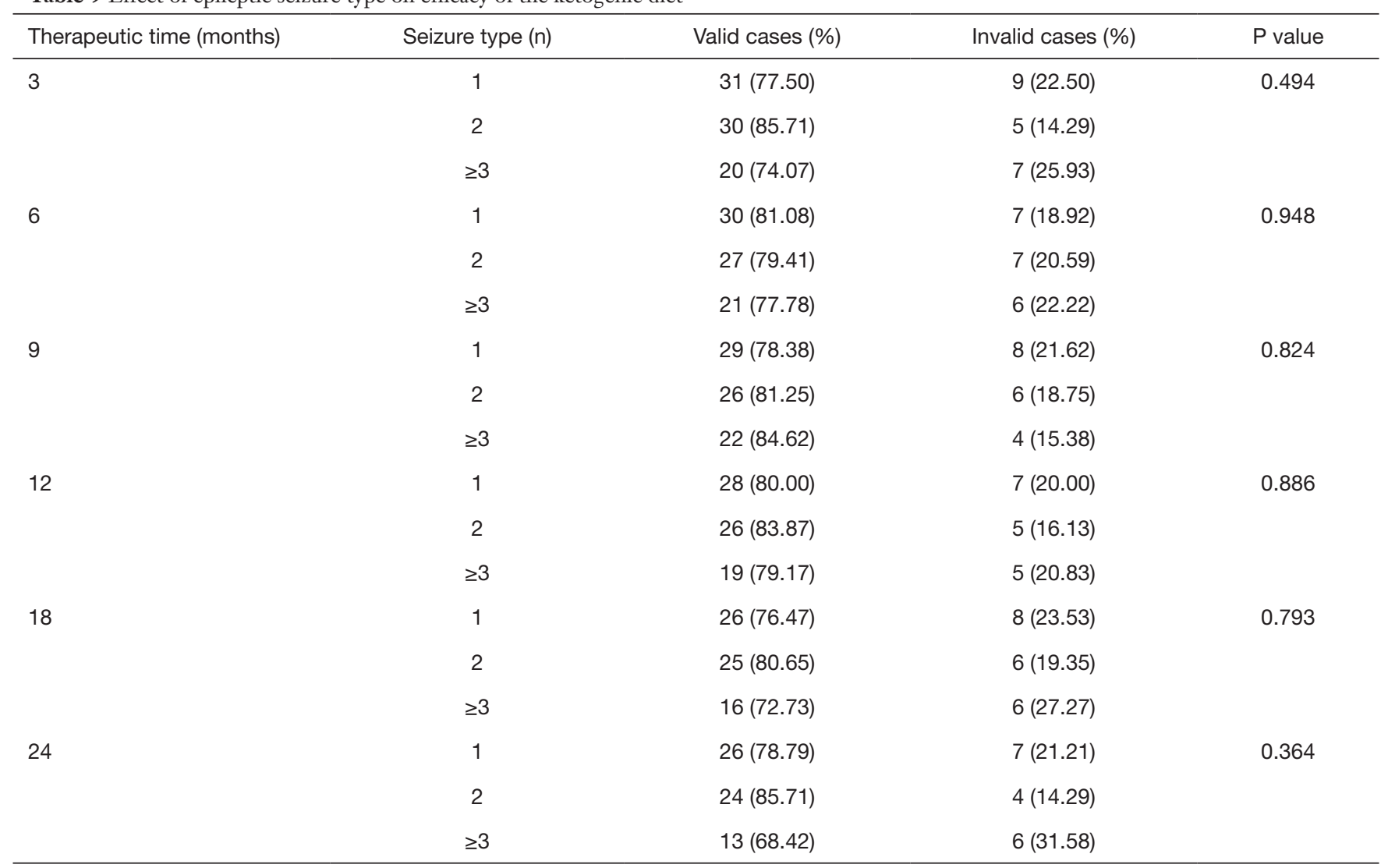

Table 10 Effects of the number of anti-epileptic drugs on efficacy of the KD

\begin{tabular}{lcccc}
\hline Therapeutic time (month) & Kinds of AEDs $(\mathrm{n})$ & Valid cases $(\%)$ & Invalid cases (\%) & P value \\
\hline 3 & $\leq 3$ & $37(80.43)$ & $9(19.57)$ & $12(21.43)$ \\
& $>3$ & $44(78.57)$ & $11(24.44)$ & 9.817 \\
6 & $\leq 3$ & $34(75.56)$ & $9(16.98)$ & $8(19.05)$ \\
9 & $>3$ & $44(83.02)$ & $10(18.87)$ \\
12 & $\leq 3$ & $34(80.95)$ & $8(20.51)$ \\
& $>3$ & $43(81.13)$ & $9(17.65)$ \\
18 & $>3$ & $31(79.49)$ & $10(27.03)$ \\
& $\leq 3$ & $42(82.35)$ & $10(20.00)$ \\
\hline
\end{tabular}


with a lower efficacy of KD treatment, which indicated that concurrent use of lamotrigine might reduce the efficacy of $\mathrm{KD}$ treatment. In our study, four children used lamotrigine, and only one child did not respond favorably to the KD, confirming the interaction between the KD and AEDs requires further study.

In conclusion, children who responded effectively at 3 months of treatment continued to benefit from the KD at later time points, which indicated it should continue when effective at 3 months. Furthermore, the results showed that the gender, age, types of epilepsy syndrome, the number of epileptic seizure types, and the number of AEDs had no significant effect on the efficacy of the KD $(\mathrm{P}>0.05)$. Therefore, the KD can be considered for children with RE in the absence of any contraindications. Finally, studies with larger sample sizes are needed to determine whether KD treatment is more effective for specific epilepsy syndrome and specific epileptic seizure type, and the interactions between the diet and AEDs.

We must point out that we did not describe the side effects of KD therapy in this study. Short-term side effects of ketogenic diets have been reported in the literature to include nausea, vomiting, hypoglycemia, acidosis, drowsiness, dehydration, and food rejection. Long-term side effects include kidney stones, constipation, growth disorders, and fractures $(20,26)$.

\section{Acknowledgments}

We thank everyone who provided help during the conduct of this study.

Funding: None.

\section{Footnote}

Reporting Checklist: The authors have completed the STROBE reporting checklist. Available at https:// tp.amegroups.com/article/view/10.21037/tp-21-595/rc

Data Sharing Statement: Available at https://tp.amegroups. com/article/view/10.21037/tp-21-595/dss

Conflicts of Interest: All authors have completed the ICMJE uniform disclosure form (available at https://tp.amegroups. com/article/view/10.21037/tp-21-595/coif). The authors have no conflicts of interest to declare.

Ethical Statement: The authors are accountable for all aspects of the work in ensuring that questions related to the accuracy or integrity of any part of the work are appropriately investigated and resolved. All procedures performed in this study involving human participants were in accordance with the Declaration of Helsinki (as revised in 2013). A prospective non-randomized controlled cohort study was used in this study and was approved by the Ethics Committee of the Children's Hospital of Chongqing Medical University [Approval document No. (2017) ethics review (research) No. 122] and informed consent was taken from the patients' guardians.

Open Access Statement: This is an Open Access article distributed in accordance with the Creative Commons Attribution-NonCommercial-NoDerivs 4.0 International License (CC BY-NC-ND 4.0), which permits the noncommercial replication and distribution of the article with the strict proviso that no changes or edits are made and the original work is properly cited (including links to both the formal publication through the relevant DOI and the license). See: https://creativecommons.org/licenses/by-nc-nd/4.0/.

\section{References}

1. Titre-Johnson S, Schoeler N, Eltze C, et al. Ketogenic diet in the treatment of epilepsy in children under the age of 2 years: study protocol for a randomised controlled trial. Trials 2017;18:195.

2. Kwan P, Arzimanoglou A, Berg AT, et al. Definition of drug resistant epilepsy: consensus proposal by the ad hoc Task Force of the ILAE Commission on Therapeutic Strategies. Epilepsia 2010;51:1069-77. Erratum in: Epilepsia. 2010;51:1922.

3. Wheless JW. History of the ketogenic diet. Epilepsia 2008;49 Suppl 8:3-5.

4. Vining EP, Freeman JM, Ballaban-Gil K, et al. A multicenter study of the efficacy of the ketogenic diet. Arch Neurol 1998;55:1433-7.

5. Martin K, Jackson CF, Levy RG, et al. Ketogenic diet and other dietary treatments for epilepsy. Cochrane Database Syst Rev 2016;2:CD001903.

6. Chen W, Kossoff EH. Long-term follow-up of children treated with the modified Atkins diet. J Child Neurol 2012;27:754-8.

7. Patel A, Pyzik PL, Turner Z, et al. Long-term outcomes of children treated with the ketogenic diet in the past. Epilepsia 2010;51:1277-82.

8. Zhu D, Wang M, Wang J, et al. Ketogenic diet effects 
on neurobehavioral development of children with intractable epilepsy: A prospective study. Epilepsy Behav 2016;55:87-91.

9. Weinshenker D. The contribution of norepinephrine and orexigenic neuropeptides to the anticonvulsant effect of the ketogenic diet. Epilepsia 2008;49 Suppl 8:104-7.

10. Wells J, Swaminathan A, Paseka J, et al. Efficacy and Safety of a Ketogenic Diet in Children and Adolescents with Refractory Epilepsy-A Review. Nutrients. 2020;12:1809.

11. Kanner AM, Ashman E, Gloss D, et al. Practice guideline update summary: Efficacy and tolerability of the new antiepileptic drugs II: Treatment-resistant epilepsy: Report of the Guideline Development, Dissemination, and Implementation Subcommittee of the American Academy of Neurology and the American Epilepsy Society. Neurology 2018;91:82-90.

12. van der Louw E, van den Hurk D, Neal E, et al. Ketogenic diet guidelines for infants with refractory epilepsy. Eur J Paediatr Neurol 2016;20:798-809.

13. Huffman J, Kossoff EH. State of the ketogenic diet(s) in epilepsy. Curr Neurol Neurosci Rep 2006;6:332-40.

14. Engel J Jr. Surgery for seizures. N Engl J Med 1996;334:647-52.

15. Alberti MJ, Agustinho A, Argumedo L, et al. Recommendations for the clinical management of children with refractory epilepsy receiving the ketogenic diet. Arch Argent Pediatr 2016;114:56-63.

16. Kossoff EH, Wang HS. Dietary therapies for epilepsy. Biomed J 2013;36:2-8.

17. Caraballo R, Vaccarezza M, Cersósimo R, et al. Long-term follow-up of the ketogenic diet for refractory epilepsy: multicenter Argentinean experience in 216 pediatric

Cite this article as: Feng L, Wang J, Li X, Hu Y, Hong S, Jiang L. Prospective control study of efficacy and influencing factors of a ketogenic diet on refractory epilepsy in children. Transl Pediatr 2022;11(1):138-148. doi: 10.21037/tp-21-595 patients. Seizure 2011;20:640-5.

18. Neal EG, Chaffe H, Schwartz RH, et al. The ketogenic diet for the treatment of childhood epilepsy: a randomised controlled trial. Lancet Neurol 2008;7:500-6.

19. Lambrechts DA, de Kinderen RJ, Vles JS, et al. A randomized controlled trial of the ketogenic diet in refractory childhood epilepsy. Acta Neurol Scand 2017;135:231-9.

20. Lima PA, Sampaio LP, Damasceno NR. Neurobiochemical mechanisms of a ketogenic diet in refractory epilepsy. Clinics (Sao Paulo) 2014;69:699-705.

21. Lutas A, Yellen G. The ketogenic diet: metabolic influences on brain excitability and epilepsy. Trends Neurosci 2013;36:32-40.

22. Kang HC, Lee YJ, Lee JS, et al. Comparison of shortversus long-term ketogenic diet for intractable infantile spasms. Epilepsia 2011;52:781-7.

23. Dhamija R, Eckert S, Wirrell E. Ketogenic diet. Can J Neurol Sci 2013;40:158-67.

24. Spilioti M, Pavlou E, Gogou M, et al. Valproate effect on ketosis in children under ketogenic diet. Eur J Paediatr Neurol 2016;20:555-9.

25. van der Louw EJ, Desadien R, Vehmeijer FO, et al. Concomitant lamotrigine use is associated with decreased efficacy of the ketogenic diet in childhood refractory epilepsy. Seizure 2015;32:75-7.

26. Freeman JM, Kossoff EH, Freeman JB, et al. The ketogenic diet: a treatment for children and others with epilepsy. New York: Demos Medical Publishing; 2007.

(English Language Editor: B. Draper) 